\title{
México y Estados Unidos frente a Centroamérica: las Conferencias de Paz de 1906 y 1907
}

Durante los primeros años del siglo $\mathrm{xx}$, los conflictos políticos entre los países centroamericanos se incrementaron. Por ello los presidentes de Estados Unidos y de México, Theodore Roosevelt y Porfirio Díaz, optaron por una política mediadora para promover la paz en la región y defender sus intereses en el istmo.

$\mathrm{E}$ ntre 1898 y 1920 , Manuel Estrada Cabrera estuvo a cargo del gobierno de Guatemala, donde mantuvo un estilo autoritario y personalista que le valió el control absoluto del país. Sin embargo, el presidente nicaragüense, José Santos Zelaya, comenzó a disputarle el poder en Centroamérica en un intento por romper el tradicional dominio guatemalteco en la región y encabezar un nuevo proyecto unionista. De esta manera, Zelaya y Estrada Cabrera se convirtieron en las dos grandes figuras políticas que se enfrentaron para imponer su hegemonía en el resto de los países del istmo.
Centroamérica era una región en la que confluían distintos intereses y, por ende, las relaciones internacionales en torno al área se definieron en función de continuas alianzas para evitar el predominio de cualquier país en el istmo. Estados Unidos quería evitar a toda costa la injerencia europea en América Central, particularmente la británica; igualmente, al gobierno estadunidense no le interesaba que México se consolidara como una potencia media en la región, mientras que éste buscaba frenar la influencia de Estados Unidos en la zona para así afianzar la propia, especialmente frente al gobierno de Guate- 
mala; los países centroamericanos no toleraban la imposición guatemalteca en sus decisiones políticas; Guatemala veía con recelo que México le disputara el poder y temía una eventual invasión, al tiempo que a Europa le preocupaba que los problemas entre México y sus vecinos del sur atrajeran la interferencia estadunidense. Por ello la contienda por la hegemonía regional conllevó continuos reacomodos y alianzas para frenar, al menos temporalmente, la explosión de los conflictos.

Al inicio del siglo $\mathrm{xx}$, la agitación política en Centroamérica llamó la atención del presidente de Estados Unidos, Theodore Roosevelt, ya que podría comprometer los intereses estadunidenses en el istmo, particularmente los trabajos para la construcción del canal interoceánico. Igualmente, el presidente de México, Porfirio Díaz, veía con preocupación los conflictos interregionales en su frontera sur ya que éstos podrían poner en peligro la estabilidad política de su país. De aquí que ambos gobiernos optaran por una política de mediación para lograr la armonía en el área, proceso que culminó con dos conferencias de paz: la primera se llevó a cabo en Costa Rica en 1906 y la segunda se realizó en Washington en 1907.

Sobre el tema de las conferencias de paz hay poco escrito y en general se han hecho breves referencias a los problemas centroamericanos de principios del siglo XX como parte de análisis más amplios de las relaciones entre México y los países del istmo y el papel de Estados Unidos en la geopolítica regional. Entre ellos destacan los trabajos de Daniel Cosío Villegas, Luis G. Zorrilla, Roberta Lajous, Jürgen Bu- chenau, Thomas D. Schoonover, Walter LaFeber y Dana G. Munro. ${ }^{1}$ El único texto dedicado exclusivamente al estudio de la participación mexicana y estadunidense para lograr la paz en Centroamérica es la tesis de maestría presentada por James William Davis en la Universidad de Texas en $1931,{ }^{2}$ que tiene como característica haberse basado casi exclusivamente en la correspondencia del Departamento de Estado, de la cual hace amplias transcripciones, y en algunos documentos de los principales acuerdos emanados de las conferencias de paz que son incluidos como apéndices al final del trabajo.

Por ello, en este artículo se pretende realizar un acercamiento al problema de la mediación de los presidentes Díaz y Roosevelt y la participación de sus agentes diplomáticos en los con. flictos entre las repúblicas de América Central en 1906 y 1907, con el objetivo de detectar los intereses de México y Estados Unidos en la región, desentrañar el complejo tejido histórico centroamericano y analizar la política de alianzas internas y externas de los gobiernos del área para poder así ofrecer un panorama más completo.

\section{El Tribunal de Arbitraje Centroamericano}

Como ya se ha mencionado, las dos grandes figuras políticas centroameri-

\footnotetext{
${ }^{1}$ Véase Cosío, Historia, 1988; Zorrilla, Relaciones, 1984; Lajous, México, 1990, t. IV; Buchenau, Sbadow, 1996; Schoonover, "Intereses", 1996, pp. 7-30; LaFeber, Inevitable, 1993; Munro, Intervention, 1964

${ }^{2}$ Davis, Joint, 1931.
} 


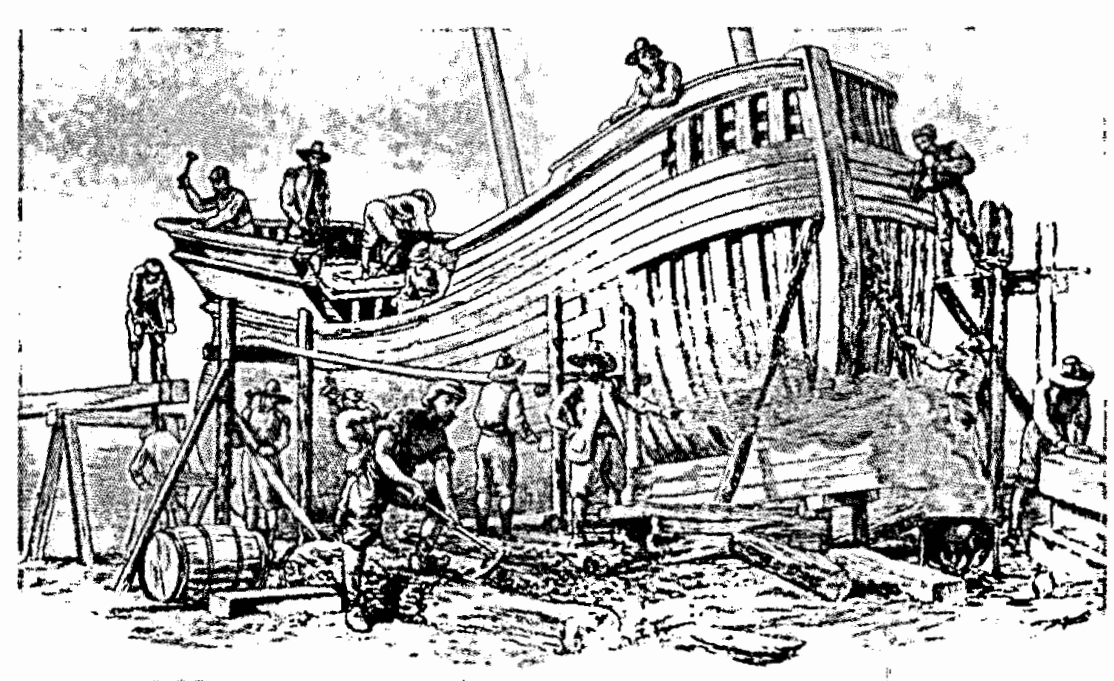

canas de la época eran Estrada Cabrera y Zelaya. Ambos deseaban alcanzar el predominio regional con el fin de negociar con Estados Unidos la construcción del canal interoceánico en condiciones ventajosas y garantizar una serie de beneficios derivados de la obra. ${ }^{3}$ Por ello, contando con una sólida base de apoyo militar, solían intervenir en los asuntos internos de los países vecinos cuyos gobiernos pudieran amenazar su estabilidad.

En 1899, Zelaya y Estrada Cabrera se aliaron para derrocar al presidente de El Salvador, Tomás Regalado, quien se oponía tajantemente a los planes unionistas de Guatemala y Nicaragua. Por lo mismo, Regalado había estrechado su relación de amistad con el gobierno mexicano, tradicionalmente contrario a

${ }^{3}$ Buchenau, Shadow, 1996, p. 52. la creación de un Estado fuerte integrado por los cinco países centroamericanos que pudiera poner en riesgo su soberanía, sobre todo si la unión la encabezaban los guatemaltecos.

De inmediato, el presidente salvadoreño solicitó la ayuda de Díaz quien ofreció mediar en el conflicto. Para ello, el representante mexicano en Centroamérica, Federico Gamboa, convocó a los cinco mandatarios de los países ístmicos a una Conferencia de Paz. Sin embargo, Nicaragua rechazó la in. vitación debido a que México se oponía a su propuesta de unión y la mediación fracasó. Asimismo, Guatemala acusó a Gamboa ante el gobierno de Washington de inmiscuirse en los asuntos centroamericanos. ${ }^{4}$ A pesar de que el conflicto no llegó a mayores debido

${ }^{4}$ Zorrilla, Relaciones, 1984 , p. 559. 
a que la alianza entre Estrada Cabrera y Zelaya era bastante frágil, el prestigio de Porfirio Díaz se vio deteriorado lo que limitó, al menos por el momento, su influencia en el área. ${ }^{5}$

En 1902 se suscitaron nuevas ten. siones entre Guatemala y El Salvador por lo que Zelaya, sintiéndose fortalecido con la idea de que el canal se construiría a través de Nicaragua, decidió convocar a una reunión de los cinco presidentes centroamericanos para tomar medidas con el fin de resolver las disputas entre ellos. Sin embargo, se sospechaba que en la junta se trataría el tema de la unión centroamericana bajo auspicios de Estados Unidos. ${ }^{6}$ Por supuesto, Estrada Cabrera se negó a asistir personalmente y sólo envió a tres militares como sus representantes. ${ }^{7}$ Los otros cuatro presidentes se reunieron en el puerto de Co. rinto y acordaron algunos puntos básicos para las relaciones interregionales durante los 20 años siguientes. El objetivo del pacto consistía en borrar los resentimientos de las diferencias pasadas y buscar mecanismos de mediación para la solución de las controversias.

Se proclamó entonces el principio del arbitraje obligatorio con la idea de someter las dificultades a un tribunal centroamericano, el cual no podría actuar sobre disputas fronterizas, para las cuales se planteaba la necesidad de con-

\footnotetext{
S Buchenau, Shadow, 1996, pp. 53-54.

${ }^{6}$ Gamboa a Mariscal, Guatemala, 21 de enero de 1902 en Archivo Histórico de la Secretaría de Relaciones Exteriores de México (en adelante AHSREM), exp. 27-4-47, pp. 9-10.

${ }^{7}$ Gamboa a Mariscal, Guatemala, 1 de febrero de 1902, en AHSREM, exp. 27-4-47, pp. 29-31.
}

vocar a árbitros extranjeros, de preferencia de nacionalidad estadunidense. Los cuatro países pidieron a Guatemala un pronunciamiento en torno a los resultados del Pacto de Corinto, a lo que Guatemala respondió manifestando su acuerdo, pero sin otorgar su firma.

Así, contando con el aval tanto de México como de Estados Unidos, el Tribunal de Arbitraje Centroamericano inició sus sesiones en el mes de octubre de 1902, en la ciudad de San José. Sin embargo, durante el año de su gestión no hubo un solo caso que arbitrar.

En 1903 se firmó un pacto semejante en San Salvador, por iniciativa del presidente José Escalón, ${ }^{8}$ en el cual se obtuvo la aceptación de Guatemala ${ }^{9}$ pero se perdió la de Costa Rica. Por medio de este acuerdo se aprobó de nueva cuenta el Pacto de Corinto, se mantuvo el principio de arbitraje obligatorio y se exigió la no intervención en los asuntos domésticos de cada estado. Los cuatro gobiernos acordaron que

en caso de surgir algún desacuerdo entre dos repúblicas, las otras dos serían los árbitros para resolver la cuestión y si la resolución de éstas no fuese satisfactoria, se determinaría oficialmente de conformidad con el Tratado de arbitraje

\footnotetext{
${ }^{8}$ Godoy al subsecretario de Relaciones Exteriores de México, Guatemala, 28 de octubre de 1903, en AHSREM, exp. 38-9-36, p. 2.

${ }^{9}$ Estrada Cabrera no tenía gran fe en los resultados de esta reunión pero aceptó participar "para demostrar su deferencia hacia las demás repúblicas hermanas". Godoy al subsecretario encargado del despacho de la Secretaría de Relaciones Exteriores de México, Guatemala, 28 de octubre de 1903, en AHSREM, exp. 38-9-36, pp. 3-7.
} 
obligatorio firmado en México en el segundo Congreso Panamericano. ${ }^{10}$

Una vez más, al año siguiente, se confirmaron los pactos anteriores y se adhirieron cuatro artículos más, los cuales aseguraban las intenciones amistosas entre los países y prometían proporcionar apoyo militar mutuo si la paz se encontrara en peligro.

Pero los problemas no tardaron en aparecer, ya que en varios estados centroamericanos había grupos de exiliados guatemaltecos organizándose para derrocar a Estrada Cabrera. Esta agitación en la región contribuyó a que el presidente Roosevelt emitiera el 6 de diciembre de 1904 su célebre corolario a la Doctrina Monroe, en el cual postulaba que las malas acciones crónicas podrían resultar en la intervención por parte de alguna nación civilizada y que, en el hemisferio occidental, Estados Unidos podría verse forzado a jugar ese papel. Por supuesto, Roosevelt deseaba evitar que los problemas de los países latinoamericanos provocaran la intervención europea en el continente para lo cual sus gobernantes debían garantizar la estabilidad política y financiera. ${ }^{11}$ Especialmente, hacía referencia al proyecto de construcción del canal de Panamá destacando que

como simple asunto de autodefensa, debemos ejercer estrecha vigilancia sobre los accesos a este canal, lo que significa que debemos estar completamente des-

${ }^{10}$ Godoy al subsecretario encargado del despacho de la Secretaría de Relaciones Exteriores de México, Guatemala, 9 de noviembre de 1903 en AHSREM, exp. 38-9-36, pp. 15-17.

${ }^{11}$ Buchenau, Shadow, 1996, pp. 58-59. piertos en cuanto a nuestros intereses en el mar Caribe. ${ }^{12}$

Obviamente, Porfirio Díaz se oponía a la intervención de las potencias europeas en la región pero tampoco estaba dispuesto a dejar el camino libre a Estados Unidos. Por ello, optó por impulsar una política exterior encaminada a mejorar su relación tanto con Roosevelt como con los mandatarios afines en el istmo centroamericano, particularmente con los de El Salvador y Nicaragua. ${ }^{13}$

De acuerdo con Thomas Schoonover, los gobiernos de México y Washington desconfiaban uno del otro. Especialmente a Díaz y a su ministro de Relaciones Exteriores, Ignacio Mariscal, les preocupaba que los países centroamericanos llegaran a unirse bajo el liderazgo de Estrada Cabrera con el apoyo estadunidense. Ambos pensaban que después de la experiencia panameña de 1903, Estados Unidos constituía una amenaza a la soberanía y la independencia de los países latinoamericanos. ${ }^{14}$ Por ello, México buscó aliarse con Nicaragua para fortalecer su postura como líder regional y decidió no dejar el camino libre a la intervención estadunidense en la región, la cual favorecería el predominio de Estrada Cabrera frente al resto de sus vecinos.

\section{LA Conferencia de PAZ dE 1906}

Dos años después, en 1906, el corolario Roosevelt fue puesto a prueba.

\footnotetext{
12 Theodore Roosevelt, "Corolario a la Doc. trina Monroe" en Núñez y Zermeño, $E U A, 1988$,

13 Buchenau, Sbadow, 1996, p. 59.

14 Schoonover, "Intereses", 1996, p. 23.
} p. 295. 
Grupos de emigrados guatemaltecos provenientes de los países contiguos llegaron a Guatemala con el fin de derrocar a Estrada Cabrera y remplazarlo por Manuel Lisandro Barillas, con el apoyo de los gobiernos de Nicaragua, El Salvador y Honduras.$^{15}$ Estrada Cabrera acusó a México de proteger a los revolucionarios de Guatemala por lo que el embajador de Estados Unidos en México, David E. Thompson, cuestionó a Ignacio Mariscal al respecto. Éste ratificó de manera tajante la posición de "estricta neutralidad" del gobierno mexicano frente a los conflictos entre los mandatarios del istmo centroamericano. ${ }^{16}$

Al ser derrotados los guatemaltecos, el salvadoreño Tomás Regalado y Zelaya acordaron que el ejército de El Salvador invadiera Guatemala en el mes de julio, ayudado por los hondureños, con el fin de destituir a Estrada Cabrera y realizar el sueño del nicaragüense de unir a las repúblicas de Centroamérica bajo su mandato. ${ }^{17} \mathrm{Sin}$ embargo, las tropas salvadoreñas fueron vencidas y Regalado fue asesinado. ${ }^{18}$

El ministro de Estados Unidos en Guatemala, Leslie Combs, personaje

${ }^{15}$ Como algunos de los atacantes se habían refugiado en México y desde ahí marcharon a Guatemala, Estrada Cabrera estaba convencido de que Porfirio Díaz les había otorgado su apoyo. Munro, Intervention, 1964, p. 144.

${ }^{16}$ Mariscal al encargado de Negocios Corrientes de la embajada en Washington y al ministro de México en Guatemala, México, 15 de junio de 1906, en AHSREM, L-E-1390, p. 58.

${ }^{17}$ Gamboa a Mariscal, Guatemala, 25 de mayo de 1906, en AHSREM, L-E-1390, p. 68.

${ }_{18}$ Zorrilla, Relaciones, 1984 , p. 561 , y LaFeber, Inevttable, 1993, p. 41. muy cercano a Estrada Cabrera, trató de mediar en la disputa pero no tuvo éxito. Además, dado que Guatemala no había firmado el Pacto de Corinto de 1902, éste no podía operar en el caso guatemalteco. Ante esta situación, Estrada Cabrera decidió aceptar la mediación de los presidentes Theodore Roosevelt y Porfirio Díaz para restablecer la paz. El primero, como ya se mencionó, deseaba evitar un conflicto bélico cerca de la zona del canal mientras que el segundo antepuso su temor de una posible guerra en Centroamérica a su rivalidad con Guatemala. ${ }^{19}$

En opinión de Cosío Villegas, a México y a Estados Unidos les interesaba contribuir a alcanzar la paz en la región por motivos diferentes. El gobierno estadunidense tenía un claro interés material para buscar el orden y la estabilidad en el istmo con el fin de lograr la penetración económica; además, su interés político consistía en demostrar el poderio y la hegemonía imperial en una zona considerada estratégica. Por su parte, México buscaba el prestigio derivado de alternar y contender con una potencia de primer orden para incrementar su papel como potencia media regional. ${ }^{20}$

En este marco, resulta significativo que a mediados del mes de julio saliera publicado un telegrama de Washington de la prensa asociada en el cual se destacaba "la mucha confianza colocada por el presidente Roosevelt y por el Departamento de Estado en el poder que tiene el presidente Díaz como jefe, de hecho, de los estados latinoame-

${ }^{19}$ Buchenau, Shadow, 1996, p. 63.

${ }^{20}$ Cosio, Historia, 1988, p. 669. 
ricanos". ${ }^{21} \mathrm{Al}$ mismo tiempo, la opinión pública de Estados Unidos tendía a exagerar las dimensiones de los movimientos subversivos en la región centroamericana con el fin de apelar a la política del Gran Garrote y legitimar la hegemonía estadunidense en el área.

En el fondo, México prefería asumir una actitud neutral ${ }^{22}$ y esperaba que Zelaya pusiera un alto a Estrada Cabrera para evitar que éste consolidara su dominio en el istmo, mientras que Estados Unidos creía que el presidente guatemalteco podría contribuir a que el mandatario de Nicaragua fuera derrocado. ${ }^{23}$ Sin embargo, sus deseos fueron dejados de lado en aras de evitar que una escalada en el conflicto pudiera lesionar sus intereses en el istmo y alterara de manera sustancial la correlación de fuerzas.

Cosío Villegas también sostiene que las potencias europeas estaban en contra de la mediación mexicana y estadunidense en los problemas de Centroamérica. En principio, no aprobaban el afán de dominio de Estados Unidos en la región y además, consideraban que lo único que estaba haciendo México era jugar un triste papel que avalaba la política exterior del gobierno de Washington. ${ }^{24}$ En el mismo sentido, Zorrilla destaca que se llegó a pensar que el presidente mexicano era utilizado por Roosevelt para que la media-

\footnotetext{
21 Intervención de Estados Unidos en la guerra centroamericana, 15 de julio de 1906, en AHSREM, L-E-1390, p. 196.

${ }^{22}$ Lajous, México, 1990, t. Iv, p. 99.

${ }^{23}$ Schoonover, "Intereses", 1996, p. 23.

${ }^{24}$ Cosío, Historia, 1988, pp. 677-678.
}

ción no pareciera una intervención unilateral de Estados Unidos. ${ }^{25}$

En todo caso, tal como afirma Jürgen Buchenau, la comediación era un arma de dos filos ya que a la vez que permitía a Díaz alcanzar sus objetivos, servía como un elemento de legitimación de la intervención de Estados Unidos en los asuntos centroamericanos. ${ }^{26}$ Incluso el gobierno de Washington se mostró ansioso por garantizar la cooperación mexicana. De aquí que el Departamento de Estado instruyera a su representante en México para que informara al presidente Díaz que Roosevelt deseaba su cooperación con el objetivo de ayudar a evitar la guerra en América Central. ${ }^{27}$ Por ello, el gobierno porfirista optó por buscar una solución diplomática con tal de impedir que los conflictos entre las repúblicas centroamericanas fueran cada día un problema mayor.

Las conversaciones para la paz se llevaron a cabo en julio de 1906 a bordo del barco Marblehead, navío de guerra estadunidense anclado frente a las costas centroamericanas. ${ }^{28} \mathrm{El}$ gobierno de Washington ofreció la cubierta de este buque de guerra "como sitio neutral donde los representantes de El Salvador y Guatemala pueden reunirse a tratar los términos del convenio efectuándose entretanto un armisticio entre los combatientes". ${ }^{29}$ Los

\footnotetext{
${ }^{25}$ Zorrilla, Relaciones, 1984, p. 562.

${ }^{26}$ Buchenau, Shadow, 1996, p. 49.

${ }^{27}$ Bacon a Thompson, Washington, $10 \mathrm{de}$ julio de 1906 en AHSREM, L-E-1390, p. 210.

${ }^{28}$ Lajous, México, 1990, t. IV, p. 100

${ }^{29}$ Bacon a Thompson, Washington, 13 de julio de 1906 en AHSREM, L-E-1390, p. 214.
} 
asistentes fueron el comisionado de Estados Unidos en Guatemala, Leslie Combs, el representante estadunidense en El Salvador, Costa Rica y Nicaragua, William L. Merry, el ministro de México para Centroamérica, Federico Gamboa, ${ }^{30}$ así como los delegados de los países centroamericanos de los cuales Nicaragua y Costa Rica participaron en calidad de observadores. ${ }^{31}$

Dos fueron los puntos álgidos que se discutieron en la reunión: el primero fue propuesto por Guatemala y exigía que los emigrados políticos fueran expulsados del país donde residían mientras que el segundo era el relativo a la obligación de someter todos los conflictos al arbitraje. Gamboa se opuso rotundamente al primero lo que conllevó un enfrentamiento directo con Combs y, en cuanto al arbitraje, propuso olvidar los conflictos pasados y limitarlo a los problemas que surgieran en el futuro. Su postura hizo que el representante estadunidense y los guatemaltecos dieran marcha atrás en sus planteamientos. ${ }^{32}$

Como resultado de las conversaciones, en las cuales Gamboa jugó un

${ }^{30}$ Éste envió a Mariscal un informe detallado del desarrollo de la reunión. Gamboa a Mariscal, Guatemala, 25 de julio de 1906, en AHSREM, L-E-1391, pp. 6-8.

${ }^{31}$ Como representantes de Guatemala asistieron Arturo Ubico, presidente del poder legislativo, José Pinto, presidente de la Suprema Corte, Juan Barrios, ministro de Relaciones y Manuel Cabral, vicepresidente de la Asamblea; por El Salvador, José Rosa Pacas, ministro de Relaciones; y en representación de Honduras, Francisco Bertrand. Cosío, Historia, 1988, p. 645.

32 Lajous, México, 1990 , t. IV, p. 100, y Zorrilla, Relaciones, 1984, p. 563. papel fundamental, se estableció la paz temporal. El tratado firmado el 20 de julio $^{33}$ exigía el cese inmediato de las hostilidades y el desarme en un plazo de ocho días, además de pedir mayor vigilancia de los gobiernos de los estados sobre los emigrados residentes en su territorio. Asimismo, en dos meses se negociaría un tratado de paz, amistad y comercio. ${ }^{34}$ Por último, las partes contratantes prometían que, en caso de nuevas dificultades, éstas serían sometidas al arbitraje de los presidentes de Estados Unidos y México. ${ }^{35}$

Quizás el logro principal fue el acuerdo de realizar una Conferencia de Paz en Costa Rica, en septiembre de $1906 .^{36}$ Los cinco estados fueron invitados, pero Nicaragua al final se negó a asistir debido a que Zelaya tenía nuevos planes para unir a Centroamérica y pensaba que eran factibles si Estados Unidos no intervenía. Para Nicaragua, el Pacto de Corinto era más que suficiente y la participación estadunidense debía ser rechazada. Según Zelaya, el convenio del Marblehead había sido impuesto por los representantes del gobierno de Washington para resolver los problemas del istmo, los cuales sólo concernían a los centroamericanos. ${ }^{37}$

En la Conferencia de San José se confirmó el Pacto de Corinto, se firmó

${ }^{33}$ Munro, Intervention, 1964, p. 145.

${ }^{34}$ Cosío, Historia, 1988, p. 648.

${ }^{35}$ Cosío Villegas señala que resultaba difícil llegar a un consenso sobre la manera de aplicar el arbitraje y si éste sería relativo a asuntos previos o sólo tendría que ver con problemas futuros. Ibid., p. 650.

${ }^{36}$ Gamboa a Mariscal, Guatemala, 21 de agosto de 1906, en AHSREM, L-E-1391, p. 145.

${ }^{37}$ Cosío Villegas, História, 1988, p. 652. 
un tratado de paz, amistad y comercio y se restableció el Tribunal de Arbitraje Centroamericano. Además, se tomaron dos acuerdos adicionales: el primero, formar una Oficina Internacional Centroamericana en la ciudad de Guatemala, la cual empezaría a funcionar en 1907 con el objetivo de preservar y estimular los intereses de Centroamérica; el segundo, crear un Instituto Pedagógico en San José al cual, si se compartían los gastos, cada estado podría mandar 20 estudiantes. Desde el punto de vista de Gamboa, "la primera es ociosa y la segunda una utopía por los términos en que se encuentran concebidas y por la poca formalidad de las altas partes contratantes". ${ }^{38}$ Ya desde ese momento, el representante mexicano consideraba sumamente difícil la creación de instituciones que garantizaran por sí solas la paz y la prosperidad en el istmo.

En cambio, en opinión del secretario de Estado, Elihu Root, los acuerdos eran fundamentales para alcanzar la paz en la región con el fin de proteger tanto la zona del canal como las inversiones de Estados Unidos en Centroamérica destinadas a la construcción de la red de ferrocarriles y al desarrollo de las plantaciones bananeras de la United Fruit Company (UFCO).$^{39}$

No obstante, los problemas en el istmo continuaron debido a que Zelaya no aceptó ni los acuerdos del Mar. blehead ni el Tratado de San José. ${ }^{40}$

${ }^{38}$ Gamboa a Mariscal, Guatemala, 17 de diciembre de 1906, en AHSREM, L-E-1391, pp. 198-199.

${ }^{39}$ Munro, Intervention, 1964, p. 146.

${ }^{40}$ Buchenau, Shadow, 1996, p. 65.
Además, de alguna manera Estrada Cabrera resultó el perdedor del conflicto pues no pudo imponerse a sus vecinos centroamericanos. Quienes se vieron fortalecidos fueron Estados Unidos y México pues, al menos temporalmente, lograron pacificar a la región. Además, con su participación, la diplomacia mexicana abrió la posibilidad de impulsar una política multilateral y pudo evitar que el gobierno de Washington asumiera el papel de garante exclusivo de la paz en el área.

\section{LA PRIMERA CONFERENCIA DE WASHINGTON}

La paz parecía estar consolidada, pero la guerra se hizo presente nuevamente en 1907. En Honduras se desató una revuelta en contra del presidente Policarpo Bonilla, quien acusó a Nicaragua de estar involucrada. Con este pretexto, los sublevados fueron perseguidos por el ejército hondureño dentro del territorio nicaragüense, matando incluso a algunos ciudadanos del país vecino. ${ }^{41}$ Ante la agresión, Zelaya pidió una indemnización, por lo que el caso fue llevado al tribunal, de acuerdo al Pacto de Corinto. Inmediatamente se ordenó el desarme, pero Nicaragua se negó y la guerra comenzó. Por su parte, Honduras, El Salvador y Costa Rica declararon roto el Pacto de 1902.

Mientras tanto, Zelaya venció al ejército hondureño, ayudó a los subleva-

${ }^{41}$ Munro sugiere la posibilidad de que Zelaya haya alentado e incluso dado su apoyo a los sublevados hondureños en contra de Bonilla. Munro, Intervention, 1964, p. 147. 
dos a deponer al presidente Bonilla e inmediatamente se preparó para invadir El Salvador. ${ }^{42}$ Esta situación una vez más alarmó a México y a Estados Unidos. El secretario de Estado, Elihu Root, intentó poner un freno a las ambiciones de Zelaya pero no pudo lograrlo. ${ }^{43}$ Por ello, el gobierno de Washington optó nuevamente por convocar a México para que de manera conjunta promovieran la paz en la región. Antes de comprometerse a participar, el ministro mexicano de Relaciones Exteriores, Ignacio Mariscal, sugirió que fueran los propios vecinos centroamericanos quienes mediaran en el conflicto, pero Guatemala, El Salvador y Costa Rica no aceptaron la propuesta. ${ }^{44}$

De aquí que los presidentes Roosevelt y Díaz decidieran invitar a los cinco estados a enviar a sus ministros a una reunión que se llevaría a cabo a finales del año 1907 y cuyo objetivo sería crear mecanismos permanentes de paz para los países del istmo. El presidente Díaz llegó a pensar que todavía era posible revivir el Pacto de Corinto, opinión que compartían los gobiernos de Honduras y Nicaragua. Sin embargo, Guatemala, El Salvador y Costa Rica optaron por la propuesta de realizar la Conferencia de Paz. ${ }^{45}$

Previo a dicho encuentro, hubo dos reuniones. En la primera, que tuvo lugar en la ciudad de Washington en el mes de septiembre, los representantes

\footnotetext{
42 Ibid.

${ }^{43}$ Incluso realizó una gira por los países centroamericanos, pero sus esfuerzos no fructificaron. Cosio, Historia, 1988, p. 672.

${ }^{44}$ Buchenau, Shadow, 1996, pp. 66-67.

45 Ibid., p. 75 .
}

de las cinco repúblicas centroamericanas discutieron un protocolo preliminar en el cual se establecía que los presidentes de Estados Unidos y de México debían "nombrar sus represen. tantes respectivos a fin de que, con carácter puramente amistoso, presten sus buenos e imparciales oficios para la realización de la conferencia". ${ }^{46}$ Además, los ministros de Guatemala, El Salvador, Costa Rica y Honduras votaron a favor de que la reunión se realizara en Washington ${ }^{47}$ y no en México, ${ }^{48}$ como Díaz hubiera deseado. Por último, se decidió por unanimidad que la conferencia se llevaría a cabo en los primeros quince días del mes de noviembre con el fin de ajustar cualquier diferencia que existiera entre las repúblicas centroamericanas. ${ }^{49}$

En la segunda reunión, ${ }^{50}$ los presidentes de Honduras, El Salvador y $\mathrm{Ni}$ caragua se encontraron en Amapala,

\footnotetext{
${ }^{46}$ Godoy a Mariscal, Washington, 11 de septiembre de 1907, en AHSREM, L-E-1392, pp. 5966.

${ }^{47} \mathrm{El}$ ministro de Nicaragua resolvió que aunque su presidente prefería que los representantes se citaran en México, estaba dispuesto a aceptar reunirse en la capital de los Estados Unidos. Godoy a Mariscal, Washington, 12 de septiembre de 1907, en AHSREM, L-E-1392, p. 34. 675.

48 Cosío Villegas, Historia, 1988, pp. 674 -

49 Godoy a Mariscal, Washington, 11 de septiembre de 1907, en AHSREM, L-E-1392, pp. 5966.

${ }^{50}$ Existía el temor entre algunos de los representantes diplomáticos en Washington, particularmente los costarricenses, de que la reunión de Amapala hubiera sido convocada con el fin secreto de hacer fracasar la Conferencia de Washington y de ello informó Godoy a Mariscal, Washington, 6 de noviembre de 1907 , en AHSREM, L-E-1394, p. 22.
} 


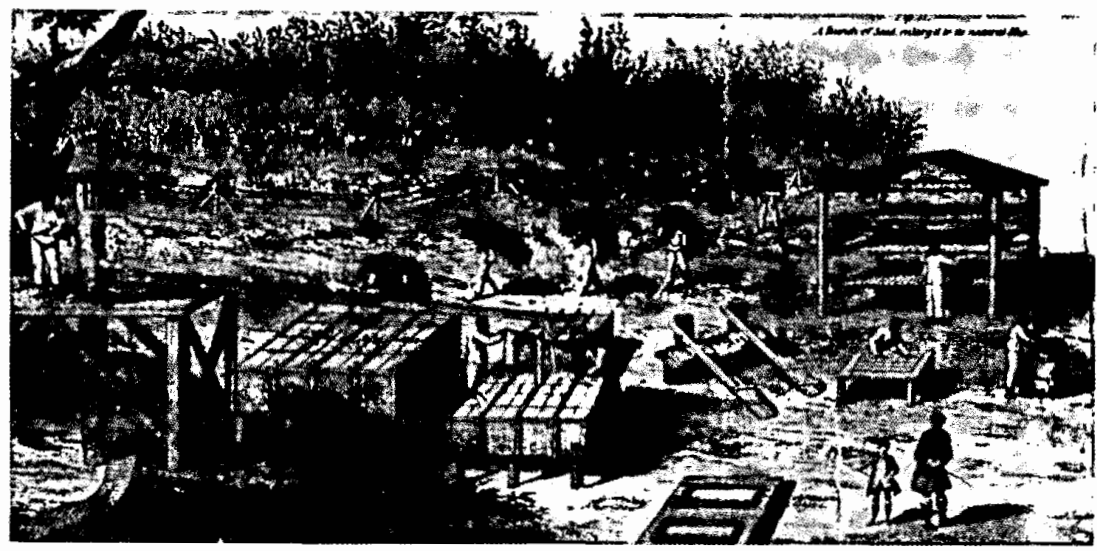

Honduras, en el mes de octubre ${ }^{51}$ y resolvieron poner fin a sus diferencias para así "establecer una armonía sólida y estable". ${ }^{52}$ Asimismo, se declararon en vigor las relaciones fraternales de los tres pueblos, se convino en comunicar a los delegados a la Conferencia de Washington los asuntos recién pactados y se decidió invitar a los presidentes de Guatemala y Costa Rica a adherirse a los trabajos en pro de la paz. ${ }^{53}$

Ello contribuyó a que en la propia conferencia de Washington que tuvo lugar en noviembre y diciembre en los salones de la Oficina Internacional de las Repúblicas Americanas continuara un ambiente en favor de un diálogo cordial. ${ }^{54}$ Por el gobierno de Estados

${ }^{51}$ Cosío, Historia, 1988, p. 676.

52 Granados a Mariscal, San Salvador, 7 de noviembre de 1907, en AHSREM, exp. 7-22-165, p. 2 .

${ }_{53} \mathrm{Ibid}$.

${ }^{54}$ Godoy a Mariscal, Washington, 1 de octubre de 1907, en AHSREM, L-E-1393, p. 100.
Unidos asistió Walter I. Buchanan y, por México, su embajador en Estados Unidos, Enrique C. Creel. ${ }^{55}$ Luis Anderson, ministro de Relaciones Exteriores de Costa Rica, fue nombrado presidente de la conferencia y fungieron como secretarios José Madriz, de Nicaragua, y Salvador Rodríguez, de El Salvador. ${ }^{56}$

Al inicio de las conversaciones, los delegados centroamericanos ${ }^{57}$ anun-

${ }^{55}$ Debido a los compromisos de Creel se decidió inaugurar la conferencia unos días después de lo planeado, el 14 de noviembre a las 2:30 de la tarde. Creel a Mariscal, Washington, 12 de noviembre de 1907, en AHSREM, LE-1394, p. 127.

${ }^{56}$ William I. Buchanan, Central, 1908, p. 5.

${ }^{57}$ Los delegados centroamericanos eran los siguientes: de Guatemala, Antonio Batres Jáuregui, Víctor Sánchez Ocana y Luis Toledo Herrarte; de El Salvador, Salvador Gallegos, Salvador Rodríguez, y Federico Mejía; de Honduras, Policarpo Bonilla, Constantino Fiallos y Ángel Ugarte; de Nicaragua, José Madriz y Luis F. Corea; de Costa Rica, Luis Anderson y Joaquín B. Calvo. Ibid., p. 4. 
ciaron que sus estados no mantendrían sus reclamos contra los otros. También fue aceptada la propuesta hondureña de otorgar amnistía plena a todos los perseguidos políticos $\mathrm{y}$, posteriormente, dicha delegación presentó un plan para unir a las cinco repúblicas como medio de terminar con las guerras. ${ }^{58}$ La tensión surgió de inmediato. Nicaragua apoyó a Honduras en su moción ${ }^{59}$ pero Costa Rica objetó que el asunto no estaba en la agenda y, aliándose con El Salvador, se pronunció en favor del plan de Guatemala de mantener la amistad sobre la base del Tratado de San José ${ }^{60}$ Finalmente, se acordó aplazar el estudio de una posible unión para otra conferencia que se realizaría un año después. ${ }^{61}$

La conferencia de Washington tuvo lugar del 14 de noviembre al 20 de diciembre de $1907 .{ }^{62}$ Después de cinco semanas de labor, el resultado fue aparentemente positivo. ${ }^{63}$ Se acordó un tratado de paz y amistad por un periodo de diez años; ${ }^{64}$ el arbitraje sobre las disputas sería obligatorio; no se permitiría a los refugiados políticos residir

\footnotetext{
${ }^{58}$ Munro, Intervention, 1964, p. 152.

59 Creel a Mariscal, Washington, 20 de noviembre de 1907 , en AHSREM, L-E-1394, pp. 210 212.

${ }^{60}$ Dichas propuestas se pueden consultar en Buchanan, Centrai, 1908, pp. 7-10.

${ }^{61}$ Godoy a Mariscal, Washington, 20 de noviembre de 1907, en AHSREM, L-E-1394, p. 134.

${ }^{62}$ Buchanan, Central, 1908, p. 3.

${ }^{63}$ Una breve sintesis de los principales logros de la Conferencia de 1907 puede ser consultada en Lockwood, Central, 1908.

${ }^{64}$ El texto completo del tratado se puede consultar en Buchanan, Central, 1908, pp. 3142.
}

en los departamentos cercanos a las fronteras cuya paz podrían hacer peligrar; se establecía la neutralidad permanente de Honduras ${ }^{65}$ y no se concedería reconocimiento a los gobiernos que obtuvieran el poder por medio de la revolución. ${ }^{66}$

Asimismo, se firmaron seis convenciones ${ }^{67}$ que especificaban la extradición para individuos culpables de crímenes, planes para un ferrocarril centroamericano, conferencias anuales de las repúblicas centroamericanas ${ }^{68}$ para los cinco años siguientes, y dos medidas tomadas del Pacto de San José: la creación tanto de la Oficina Centroamericana de Guatemala como del Instituto Pedagógico en San José. La última convención, punto nodal de todo el plan, proponía la creación de una Corte de Justicia Centroamericana. ${ }^{69}$ Para muchos, éste era el único camino para llegar al ideal de la confederación centroamericana, tomando la paz como el paso previo e indispensable para alcanzar la unión.

\footnotetext{
${ }^{65}$ Debido a la posición estratégica de Honduras dentro del istmo aunada a su debilidad política, tradicionalmente había sido usada por otros países para sus propios fines involucrándola en los conflictos regionales. Munro, Intervention, 1964, p. 153.

${ }^{66}$ Zorrilla, Relaciones, 1984 , p. 565.

${ }^{67}$ Las seis convenciones eran de comunicación, de extradición, para el establecimiento de un Instituto Pedagógico Centroamericano, de una Oficina Internacional Centroamericana, sobre futuras conferencias centroamericanas y adicional al tratado general. Creel a Mariscal, Washington, 28 de diciembre de 1907 , en AHSREM, L-E-1394, pp. 22-23. Los textos completos de las Convenciones se pueden consultar en Buchanan, Central, 1908, pp. 43-80.

${ }^{68}$ Cosío, Historia, 1988, p. 677.

${ }^{69}$ Lajous, México, 1990, t. Iv, p. 102.
} 
Precisamente M. Francis Rey, Jefe de Conferencias de la Facultad de Derecho de París, elaboró en 1911 un trabajo $^{70}$ en el que desarrollaba la idea de que las instituciones emanadas de los tratados de 1907 tendían claramente a allanar el camino para alcanzar la unión centroamericana. No obstante, remarcaba la necesidad de

disipar las desconfianzas y extinguir las rivalidades que existen entre los estados del istmo, desarrollar sus comunes intereses, aumentar sus relaciones comerciales, hacer que en los pueblos nazca el sentimiento de una patria común, superior a la patria de origen, y trabajar en fin porque en los espíritus penetre la idea de que el respeto a la ley está por encima de los intereses de partido. $^{71}$

En la mente de los centroamericanos de la época, la unión continuaba siendo un ideal alcanzable.

De acuerdo con Buchenau, a diferencia de la Conferencia de San José, México jugó un papel secundario en Washington ya que Creel permitió que Buchanan encabezara la reunión. De aquí que Estados Unidos consiguiera sus objetivos principales: derrotar la propuesta de Zelaya para unificar a Centroamérica y lograr la estabilidad regional sin necesidad de intervenir continuamente. Por su parte, México vio con buenos ojos la creación de una serie de mecanismos de arbitraje propios de los países centroamericanos los cuales les permitirían resolver sus diferencias sin que fuera necesaria la

${ }^{70}$ Rey, Unión, 1911.

${ }^{71}$ Ibid., p. 7. intromisión del gobierno de Washington en el área. ${ }^{72}$

\section{A MANERA DE CONCLUSIÓN}

Los constantes conflictos en la región centroamericana durante los primeros años del siglo XX tuvieron su origen en las pugnas derivadas de los intentos unionistas; en los ataques entre liberales y conservadores dentro de cada uno de los países del istmo; en los frecuentes intentos por derrocar a los gobiernos, tanto por opositores internos como por externos; en la actividad subversiva de los refugiados políticos quienes desde un país vecino y a veces con el apoyo de su gobierno intentaban desestabilizar al régimen del cual habían huido.

Estados Unidos buscaba la paz en Centroamérica con el fin de proteger tanto sus intereses canaleros como las inversiones de capital privado en la región y evitar la injerencia externa en el istmo, principalmente de las potencias europeas y de México. Por su parte, Porfirio Díaz buscó desarrollar una política exterior activa para frenar la intervención estadunidense en el istmo, al tiempo que intentaba garantizar la paz con el fin de evitar cualquier posible conflicto en su frontera sur. De aquí que ambos buscaran a los gobiernos afines en la región para establecer una política de alianzas sólida. Así, Washington encontró en el mandatario guatemalteco a un fuerte aliado para lograr sus fines mientras que la diploma-

${ }^{72}$ Buchenau, Shadow, 1996, pp. 76-77. 
cia mexicana contó con el apoyo fundamental de los nicaragüenses.

Debido a que las instancias locales de arbitraje habían comprobado su falta de eficacia, ambos países optaron por la mediación conjunta ya que a los dos les convenía que el otro participara en el proceso de paz para así controlarse mutuamente. Al principio Estados Unidos desconfiaba de la participación mexicana en los asuntos de la política interna centroamericana, pero después prefirieró tenerlo como aliado para aprovechar su influencia en la región y para presionarlo con el fin de que mejorara su relación con el gobierno de Estrada Cabrera. A su vez, Díaz no deseaba ser utilizado por Roosevelt pero prefirió cooperar con él para evitar cualquier posible intervención militar estadunidense en el istmo y, de paso, impedir que Estrada Cabrera se consolidara como líder de los países centroamericanos con el apoyo de Estados Unidos.

A partir de las conferencias de paz se crearon nuevos mecanismos de conciliación pero sus resultados fueron bastante limitados. Además, se comprobó que las instancias locales para la paz no eran ajenas a la política internacional y, en particular, a los designios que Estados Unidos había establecido para el istmo centroamericano en función de sus intereses estratégicos.

\section{Bibliografía}

-Buchanan, William I., The Central American Peace Conference beld at Washington,
D.C. 1907, Government Printing Office, Washington, 1908.

-Buchenau, Jürgen, In the shadow of the giant. The making of Mexico's Central America policy, 1876-1930, The Alabama University Press, Tuscaloosa, 1996.

-Cosío Villegas, Daniel, Historia Moderna de México. El porfiriato. Vida política exterior, Editorial Hermes, México, 1988.

-Davis, James William, The joint intervention of the United States and Mexico in Central America in 1906 and 1907, University of Texas, Austin, 1931.

-LaFeber, Walter, Inevitable revolutions. The United States in Central America, W. W. Norton and Company, Nueva York, 1993.

-Lajous, Roberta, México y el mundo. Historia de sus relaciones exteriores, Senado de la República, México, 1990, 8 vols.

-Lockwood, Belva Ann Bennett, The Central American peace congress and international arbitration court for the five Central American republics, s.e., Washington, 1908.

-Munro, Dana G., Intervention and dollar diplomacy in the Caribbean, 1900. 1921, Princeton University Press, Nueva Jersey, 1964.

-Núñez, Silvia y Guillermo Zermeño (comps.), EUA: Documentos de su bistoria política III, Instituto Mora, México, 1988.

-Rey, Francis, La unión centroamericana. Estudio relativo a las instituciones creadas en Washington por la Conferencia de Paz centroamericana de 1907, Tipografia Sánchez y de Guise, Guatemala, 1911.

-Schoonover, Thomas D., "Los intereses europeos y estadunidenses en las relaciones políticas México-Guatemala", Secuencia. Revista de Historia y Ciencias Sociales, Instituto Mora, núm. 34, 1996, pp. 7-30, México.

-Zorrilla, Luis G., Relaciones de México con la República de Centro América y con Guatemala, Porrúa, México, 1984. 